University of Wollongong

Research Online

Faculty of Engineering - Papers (Archive)

Faculty of Engineering and Information

Sciences

$15-2-1997$

\title{
Nonlinear transport in steady-state terahertz-driven two-dimensional electron gases
}

W. Xu

University of Wollongong

C. Zhang

University of Wollongong, czhang@uow.edu.au

Follow this and additional works at: https://ro.uow.edu.au/engpapers

Part of the Engineering Commons

https://ro.uow.edu.au/engpapers/251

\section{Recommended Citation}

$\mathrm{Xu}, \mathrm{W}$. and Zhang, C.: Nonlinear transport in steady-state terahertz-driven two-dimensional electron gases 1997.

https://ro.uow.edu.au/engpapers/251

Research Online is the open access institutional repository for the University of Wollongong. For further information contact the UOW Library: research-pubs@uow.edu.au 


\title{
Nonlinear transport in steady-state terahertz-driven two-dimensional electron gases
}

\author{
W. $\mathrm{Xu}^{*}$ and C. Zhang \\ Department of Physics, University of Wollongong, Wollongong, NSW 2522, Australia \\ (Received 19 September 1996; revised manuscript received 4 November 1996)
}

\begin{abstract}
We present a detailed theoretical study on nonlinear transport and optical properties in two-dimensional semiconductor systems (2DSS's) subjected to intense terahertz electromagnetic fields. By solving the momentum- and energy-balance equations using a steady-state Boltzmann equation approach, where electron interaction with LO phonons is taken into account under the lowest-order approximation for GaAs-based 2DSS's, we have investigated the dependence of electron temperature, momentum relaxation time, and conductivity on the intensity and frequency of $\mathrm{THz}$ radiation fields. The results obtained from this study are in line with those obtained from recent experimental measurements [N. G. Asmar et al., Appl. Phys. Lett. 68, 829 (1996)]. [S0163-1829(97)04308-7]
\end{abstract}

\section{INTRODUCTION}

With the development and application of state-of-the-art techniques such as free-electron lasers (FEL's), it has become realistic to investigate the transport and optical properties of a device under terahertz $(\mathrm{THz})$ electromagnetic radiations. FEL's can provide the source of the linearly polarized intense $\mathrm{THz}$ radiation which is of significant impact on the study of novel condensed-matter materials such as two-dimensional semiconductor systems (2DSS's). For a 2DSS grown by using techniques such as molecular beam epitaxy, the conducting electrons are confined within the nanometer distance scale so that the energies (e.g., electronic subband energy, electron kinetic energy, Fermi energy, etc.) are on the meV scale. Noting that an energy $\hbar \omega \sim \mathrm{meV}$ corresponds to a frequency $\omega \sim \mathrm{THz}, \mathrm{THz}$ electromagnetic waves may couple strongly to the 2DSS's, which gives us a possibility to observe photon-induced quantum resonance effects such as magneto-photon-phonon resonances. ${ }^{1}$ Furthermore, for GaAs-based 2DSS's the rate of electronic transitions via electron interactions with impurities and phonons may be on the scale of $\mathrm{THz}$ (i.e., $10^{12} \mathrm{~s}^{-1}$ ). ${ }^{2,3} \mathrm{THz}$ electromagnetic radiation will modify strongly the processes of momentum and energy relaxation for excited electrons in the structure, which implies that GaAs-based 2DSS's can be applied as $\mathrm{THz}$ devices working in the linear and nonlinear response regimes.

Recently, experimental measurements have been carried out ${ }^{4}$ by Asmar et al. in studying the nonlinear transport and optical properties in intense THz-driven 2DSS's. Using photoluminescence (PL) and transport measurements, they have determined the dependence of electron temperature in $\mathrm{Al}_{x} \mathrm{Ga}_{1-x} \mathrm{As} / \mathrm{GaAs}$ quantum wells on the intensity and frequency of THz radiations provided by the FEL's. The results obtained from these measurements have indicated that under strong $\mathrm{THz}$ radiations, (i) electron energy distribution can be characterized by an effective electron temperature; (ii) electron-LO-phonon interaction dominates both momentum and energy relaxation; (iii) radiation frequency has a strong influence on the electron temperature; and (iv) hot-phonon effects such as phonon drift are negligible. This initial work has shed light on the interesting features of nonlinear trans- port and optical effects in $\mathrm{Al}_{x} \mathrm{Ga}_{1-x} \mathrm{As} / \mathrm{GaAs}$-based 2DSS's in $\mathrm{THz}$ electromagnetic fields, and impelled further theoretical study.

In contrast to the experimental measurements carried out only recently in the $\mathrm{THz}$ regime, ${ }^{4,5}$ there exist a number of theoretical studies on high-frequency ac response of semiconductor materials. Important contributions have been made by some authors. ${ }^{6-13}$ However, these investigations focused either on the linear response, ${ }^{6-9}$ or on the situation with a weak ac field under a strong dc bias, ${ }^{10,12}$ or on the problems of the $v-E$ relation $^{13}$ and thermal-noise temperature. ${ }^{11}$ Further, in most theoretical study on nonlinear ac response in semiconductors one normally took the electron temperature as an input parameter to calculate quantities such as conductivity, memory function, etc. ${ }^{2,10,11}$ Therefore the need for a theoretical study in conjunction with the experiments conducted by Ref. 4 is evident. The motivation of this study is to attempt a contribution in this direction. In this paper a detailed consideration and derivation of the model calculation is presented in Sec. II. The numerical results for an $\mathrm{Al}_{x} \mathrm{Ga}_{1-x} \mathrm{As} / \mathrm{GaAs}$ heterojunction subjected to $\mathrm{THz}$ radiations are shown and discussed in Sec. III. The present study is summarized in Sec. IV.

\section{MODEL}

To set up a tractable method to study transport and optical properties of a two-dimensional electron gas (2DEG), especially in the nonlinear response regime, has been an outstanding problem. It is well known that the balance equation approach is a powerful tool in studying the nonlinear response of an electronic device to the dc and ac fields applied. In a pioneering work, ${ }^{14}$ Thornber and Feynman (TF) derived a momentum-balance equation to determine the nonlinear $v-E$ relation in a strong polar semiconductor by using path integrals. Peeters and Devreese have shown ${ }^{15}$ that the electron distribution in the approach of the TF theory was essentially a displaced Maxwellian. This implies that statistical distribution functions such as Maxwellian and Fermi-Dirac functions can be used to describe the electron distribution in the nonlinear response regime. Based on the TF theory, Lei and Ting also proposed ${ }^{16}$ balance equations which were de- 
rived under a non-Boltzmann approach. Later, Lei and Horing generated these balance equations for the case where an ac driving field is present. ${ }^{11}$ The momentum- and energybalance equations proposed by Lei and co-workers are derived from applying a Green's function approach to the TF theory and starting from the Liouville equation for the density matrix. To employ these balance equations, one has to solve the problem of (i) the correlation functions, which do not offer simple analytic results; and (ii) the frictional force and energy transfer rate, of which both are very complicated functional forms, especially in the presence of an ac field and for the energy-balance equation. ${ }^{11}$ In this paper we attempt to make our model as simple as possible in order to understand the most important physical mechanism responsible for the problem and to make the physical considerations more transparent. We derive the balance equations from the Boltzmann equation. With this approach, one may detour the difficulties to solve directly the Boltzmann equation and keep the main merits of the Boltzmann equation to describe the response of electrons to the applied fields. We have successfully applied this approach to the problem of hot-electron transport in 2DSS's in strong dc electric fields. ${ }^{17}$ In the present study we generate this approach for the case where the electronic device is driven by an ac field.

\section{A. Momentum- and energy-balance equations for a 2DEG}

In the presence of an electromagnetic radiation field, the time-dependent steady-state Boltzmann equation for a $2 \mathrm{DEG}$ is given by

$$
\frac{\partial f_{n}(\mathbf{k}, t)}{\partial t}+\frac{\mathbf{F}}{\hbar} \cdot \nabla_{\mathbf{k}} f_{n}(\mathbf{k}, t)=I_{n}(\mathbf{k}, t),
$$

where $f_{n}(\mathbf{k}, t)$ is the momentum distribution function for an electron in a state $|\mathbf{k}, n\rangle$ at time $t, \mathbf{k}=\left(k_{x}, k_{y}\right)$ is the wave vector of the electron, $\mathbf{F}=\mathbf{F}(t)$ is the force acting on the electron, and the contribution from scattering in the case of nondegenerate statistics is given by

$$
\begin{aligned}
I_{n}(\mathbf{k}, t)= & g_{s} \sum_{n^{\prime}, \mathbf{k}^{\prime}}\left[f_{n^{\prime}}\left(\mathbf{k}^{\prime}, t\right) W_{n n^{\prime}}\left(\mathbf{k}, \mathbf{k}^{\prime}\right)\right. \\
& \left.-f_{n}(\mathbf{k}, t) W_{n^{\prime} n}\left(\mathbf{k}^{\prime}, \mathbf{k}\right)\right],
\end{aligned}
$$

with $g_{s}=2$ for spin degeneracy and $W_{n^{\prime}{ }_{n}}\left(\mathbf{k}^{\prime}, \mathbf{k}\right)$ being the steady-state transition rate for an electron scattered from a state $|\mathbf{k}, n\rangle$ to a state $\left|\mathbf{k}^{\prime}, n^{\prime}\right\rangle$.

The effect of the electromagnetic field with a frequency $\omega$ can be represented by an ac electric field, which results in $\mathbf{F}=-e \mathbf{E}$, where $\mathbf{E}=(E, 0,0)$ and $E=E(t)=E_{0} e^{-i \omega t}$. Here (i) we have considered a situation where the electromagnetic radiation is polarized along the $2 \mathrm{D}$ plane of a $2 \mathrm{DEG}$ (taken along the $x$ direction); (ii) in this configuration, the effects of direct optical absorption via intersubband electronic transitions can be ignored; and (iii) the effect of the presence of a dc electric field is not taken into account in this study. We assume that $E_{\mathrm{ac}} \gg E_{\mathrm{dc}}$ as was realized in the experiments conducted in Refs. 4 and 5. Corresponding to the ac electric field applied, the average velocity of electrons can be taken as $\mathbf{v}=(v, 0,0)$ with $v=v(t)=v_{0} e^{-i \omega t}$. It can be justified that $v(t)=v_{0} e^{-i \omega t}$ is the steady-state solution of the Boltzmann equation for the driving field $E(t)=E_{0} e^{-i \omega t}$. Using the bal- ance equation approach, the idea is to choose a certain functional form for the electron distribution function (EDF), of which the drifted Maxwellian is the most popularly used statistical energy distribution function for a system with lower electron density and at relatively high temperature or high electron temperature. ${ }^{17}$ Thus we have $f_{n}(\mathbf{k}, t)=f\left[E_{n}\left(k_{x}\right.\right.$ $\left.\left.-m^{*} v / \hbar, k_{y}\right)\right]$ drifted by electron velocity, where $f(x)$ $=e^{-x / k_{B} T_{e}}$ with $T_{e}$ being the electron temperature, and $E_{n}\left(k_{x}, k_{y}\right)=E_{n}(\mathbf{k})=\epsilon_{n}+\hbar^{2}\left(k_{x}^{2}+k_{y}^{2}\right) / 2 m^{*}$ is the energy spectrum of a $2 \mathrm{DEG}$ with $\epsilon_{n}$ the energy of the $n$th electronic subband and $m^{*}$ the effective electron mass. After taking into account the above remarks, we can derive balance equations starting from the Boltzmann equation.

When $f_{n}(\mathbf{k}, t)=f\left[E_{n}\left(k_{x}-m^{*} v / \hbar, k_{y}\right)\right]$, we have

$$
\frac{\partial f_{n}(\mathbf{k}, t)}{\partial t}=i \hbar \omega v\left(k_{x}-\frac{m^{*} v}{\hbar}\right) \frac{\partial f\left(\epsilon_{n}+\mathcal{E}\right)}{\partial \mathcal{E}},
$$

and

$$
\frac{\partial f_{n}(\mathbf{k}, t)}{\partial k_{x}}=\frac{\hbar^{2}}{m^{*}}\left(k_{x}-\frac{m^{*} v}{\hbar}\right) \frac{\partial f\left(\boldsymbol{\epsilon}_{n}+\mathcal{E}\right)}{\partial \mathcal{E}},
$$

where $\mathcal{E}=\hbar^{2}\left[\left(k_{x}-m^{*} v / \hbar\right)^{2}+k_{y}^{2}\right] / 2 m^{*}$. Thus we obtain

$$
\begin{aligned}
& \int d^{2} \mathbf{k} \frac{\partial f_{n}(\mathbf{k}, t)}{\partial t}=\int d^{2} \mathbf{k} \frac{\partial f_{n}(\mathbf{k}, t)}{\partial k_{x}} \\
&=\int d^{2} \mathbf{k} k_{y} \frac{\partial f_{n}(\mathbf{k}, t)}{\partial t} \\
&=\int d^{2} \mathbf{k} k_{y} \frac{\partial f_{n}\left(\mathbf{k}, k_{x}\right)}{\partial k_{x}} \\
&=\int d^{2} \mathbf{k} k_{x} k_{y} \frac{\partial f_{n}(\mathbf{k}, t)}{\partial t} \\
&=\int d^{2} \mathbf{k} k_{x} k_{y} \frac{\partial f_{n}(\mathbf{k}, t)}{\partial k_{x}}=0 \\
& \sum_{n} \int d^{2} \mathbf{k} k_{x} \frac{\partial f_{n}(\mathbf{k}, t)}{\partial t}=-i \omega \frac{2 \pi^{2} n_{e} m^{*}}{\hbar} v \\
& \sum_{n} \int d^{2} \mathbf{k} k_{x} \frac{\partial f_{n}(\mathbf{k}, t)}{\partial k_{x}}=-2 \pi^{2} n_{e} \\
& \sum_{n} \int d^{2} \mathbf{k} E_{n}(\mathbf{k}) \frac{\partial f_{n}(\mathbf{k}, t)}{\partial t}=-i \omega 2 \pi^{2} n_{e} m^{*} v^{2}
\end{aligned}
$$

and

$$
\sum_{n} \int d^{2} \mathbf{k} E_{n}(\mathbf{k}) \frac{\partial f_{n}(\mathbf{k}, t)}{\partial k_{x}}=-2 \pi^{2} \hbar n_{e} v .
$$

Here we have used a condition of electron number conservation, i.e., after using the density of states for a 2DEG, we have $n_{e}=\left(m^{*} / \pi \hbar^{2}\right) \Sigma_{n} \int_{0}^{\infty} d E f\left(E+\epsilon_{n}\right)$, where $n_{e}$ is the electron density of the 2DEG.

From Eq. (3a), we see that the mass equation (multiplying $\Sigma_{n, \mathbf{k}}$ to both sides of the Boltzmann equation) has been balanced by the EDF chosen above. At the first moment, the momentum-balance equation can be derived by multiplying 
$\Sigma_{n, \mathbf{k}} \mathbf{k}$ to both sides of the Boltzmann equation. Using Eqs. (3b) and (3c), we obtained the conductivity

$$
\sigma=\frac{n_{e} e^{2}}{m^{*}} \frac{\tau}{1-i \omega \tau},
$$

where $\sigma=-n_{e} e v / E$ and the momentum relaxation time $\tau$ is given by

$$
\begin{aligned}
\frac{1}{\tau} \simeq & \frac{\hbar^{2}}{4 \pi^{4} n_{e} m^{*}} \sum_{n^{\prime}, n} \int d^{2} \mathbf{k} d^{2} \mathbf{k}^{\prime} k_{x}\left(k_{x}^{\prime}-k_{x}\right) \\
& \times \frac{\partial f\left(\boldsymbol{\epsilon}_{n}+x\right)}{\partial x} W_{n^{\prime} n}\left(\mathbf{k}^{\prime}, \mathbf{k}\right),
\end{aligned}
$$

with $x=\hbar^{2} k^{2} / 2 m^{*}$. The conductivity shown by Eq. (4a) is in the form of the Drude formula. At the second moment, the energy-balance equation can be derived by multiplying $\sum_{n, \mathbf{k}} E_{n}(\mathbf{k})$ to both sides of the Boltzmann equation. After using Eqs. (3d) and (3e), we have

$$
\begin{aligned}
\sigma E^{2} \simeq & -\frac{1-i \omega \tau}{4 \pi^{4}} \sum_{n^{\prime}, n} \int d^{2} \mathbf{k} d^{2} \mathbf{k}^{\prime}\left[E_{n^{\prime}}\left(\mathbf{k}^{\prime}\right)-E_{n}(\mathbf{k})\right] \\
& \times f\left(\epsilon_{n}+x\right) W_{n^{\prime} n}\left(\mathbf{k}^{\prime}, \mathbf{k}\right) .
\end{aligned}
$$

In the derivation of Eqs. (4) and (5), we have used the approximation that in the presence of intense electromagnetic radiation and for a zero or weak dc electric field applied, the average electron velocity $v$ is very small in comparison with the electron wave vector $k_{x}$ so that $\hbar v k_{x} \ll k_{B} T_{e}$. This approximation results in

$$
f_{n}(\mathbf{k}, t) \simeq f\left(\epsilon_{n}+x\right)-\hbar v k_{x} \frac{\partial f\left(\epsilon_{n}+x\right)}{\partial x} .
$$

In the momentum-balance equation, the usage of the approximation given by Eq. (6) is equivalent to the relaxation time approximation but now the relaxation time depends on the electron temperature.

We note that at a steady state and using the momentumand energy-balance equation approach, we do not need to do time average in the Boltzmann equation. The physical reason behind this is that we have used a steady-state solution of the electron velocity and a steady-state electronic transition rate derived at the limit $t \rightarrow \infty$.

\section{B. For the case of electron-LO-phonon interactions}

In the presence of strong driving fields, the electrons in the system will be heated and the electron-LO-phonon interaction is the principal channel for relaxation of excited electrons in polar semiconductors such as GaAs. After applying the Fröhlich Hamiltonian to Fermi's golden rule, the steady-state transition rate induced by electron interactions with LO phonons and with radiation fields polarized along the $2 \mathrm{D}$ plane in a $2 \mathrm{DEG}$ is given by

$$
W_{n^{\prime} n}\left(\mathbf{k}^{\prime}, \mathbf{k}\right)=W_{n^{\prime} n}^{+}\left(\mathbf{k}^{\prime}, \mathbf{k}\right)+W_{n^{\prime} n}^{-}\left(\mathbf{k}^{\prime}, \mathbf{k}\right)
$$

$$
\begin{aligned}
W_{n^{\prime} n}^{ \pm}\left(\mathbf{k}^{\prime}, \mathbf{k}\right)= & 4 \pi \alpha L_{0} \hbar \omega_{\mathrm{LO}}^{2}\left[\begin{array}{c}
N_{0} \\
N_{0}+1
\end{array}\right] X_{n^{\prime} n}(q) \delta_{\mathbf{k}^{\prime}, \mathbf{k}+\mathbf{q}} \\
& \times \sum_{m=0} J_{m}^{2}(\mathcal{A}) \\
& \times \delta\left[E_{n^{\prime}}\left(\mathbf{k}^{\prime}\right)-E_{n}(\mathbf{k}) \mp \hbar \omega_{\mathrm{LO}}-m \hbar \omega\right]
\end{aligned}
$$

where the sign $+(-)$ refers to absorption (emission) of a LO phonon with an energy $\hbar \omega_{\mathrm{LO}}, N_{0}=\left(e^{\hbar \omega_{\mathrm{LO}} / k_{B} T}-1\right)^{-1}$ is the LO-phonon occupation number, $\mathbf{Q}=\left(\mathbf{q}, q_{z}\right)=\left(q_{x}, q_{y}, q_{z}\right)$ the phonon wave vector, $\alpha$ the electron-LO-phonon coupling constant, $L_{0}=\left(\hbar / 2 m^{*} \omega_{\mathrm{LO}}\right)^{1 / 2}$ the polaron radius, $X_{n^{\prime} n}(q)=\int_{-\infty}^{\infty} d q_{z}\left|G_{n^{\prime}} n\left(q_{z}\right)\right|^{2} /\left(q^{2}+q_{z}^{2}\right)$ with $G_{n^{\prime} n}\left(q_{z}\right)$ $=\left\langle n^{\prime}\left|e^{i q_{z} z}\right| n\right\rangle$ being the form factor for electron-phonon interaction, and $J_{m}(x)$ is the Bessel function. Further, $\mathcal{A}=\left(\bar{e} E_{0} / m^{*} \omega^{2}\right) \mathbf{u} \cdot \mathbf{q}$, where $\mathbf{u}$ is the polarization vector of the radiation. Here, only the process of optical absorption is included, and the scattering induced by direct electronphoton interactions has been ignored (because the radiation field is polarized along the 2D plane).

From Eq. (7), we see that the coupled electron-photonphonon interactions come from the higher-order contributions, i.e., from $m \geqslant 1$ (corresponding to the $m$-photon absorptions). From the fact that in polar semiconductors such as GaAs the electrons interact much more strongly with phonons than with photons, the contribution to the conductivity from the electron-photon-phonon interactions is much smaller than that from the electron-phonon interactions (i.e., the term depends on $m=0$ ). The main physical reason behind this is that in polar semiconductors, there exists a very strong polar electric field $F_{0}=\omega_{\mathrm{LO}}\left(2 m * \hbar \omega_{\mathrm{LO}}\right)^{1 / 2} / e$, for example, for GaAs $F_{0}$ is about $100 \mathrm{kV} / \mathrm{cm}$. Moreover, under the highfrequency electromagnetic radiations (e.g., $\mathrm{THz}$ or far infrared) and for electron interactions with long-wavelength phonon modes such as LO phonons, $\mathcal{A} \sim E_{0} q \omega^{-2}$ may be small even at strong radiation fields. In the present study, we ignore the higher-order interactions (i.e., taking only $m=0$ ) and use the high-frequency (large $\omega$ ) and long-wavelength phonon (small $q_{x}$ ) approximations to the interactions [i.e., $\left.J_{0}(\mathcal{A} \rightarrow 0)=1\right]$. Thus the electronic transition rate is isotropic so that the conductivity becomes isotropic: $\sigma_{x x}=\sigma_{y y}=\sigma$ and $\sigma_{x y}=\sigma_{y x}=0$. For semiconductor materials such as $\mathrm{Si}$ in which the electron-phonon interactions are relatively weak, an anisotropic conductivity has been predicted recently ${ }^{9}$ at low-frequency (microwave) irradiations.

For studying optical and transport properties of a device driven by an electromagnetic field, two quantities, the real parts of the conductivity and the electron-energy-loss rate (EELR), are connected to the optical absorption coefficient and the conductivity (or mobility) measured experimentally. Introducing the transition rate for electron-LO-phonon scattering under the leading-order and high-frequency approximations into the momentum-balance equation, we have

$$
\operatorname{Re} \sigma=\frac{n_{e} e^{2}}{m^{*}} \frac{\tau}{1+(\omega \tau)^{2}},
$$

where the momentum relaxation time is given by $1 / \tau$ $=1 / \tau^{+}+1 / \tau^{-}$and 


$$
\begin{aligned}
\frac{1}{\tau^{ \pm}}= & \frac{\alpha L_{0} \omega_{\mathrm{LO}}^{2} m^{* 2}}{\pi \hbar^{3} n_{e} \sqrt{\pi k_{B} T_{e}}}\left[\begin{array}{c}
N_{0} \\
N_{0}+1
\end{array}\right] \sum_{n^{\prime}, n} \int_{0}^{\infty} \frac{d y}{\sqrt{y}} e^{-A_{n^{\prime} n}^{ \pm}(y) / k_{B} T_{e}} \\
& \times\left(\epsilon_{n^{\prime}}-\epsilon_{n} \mp \hbar \omega_{\mathrm{LO}}+y\right) X_{n^{\prime} n}\left[\left(\frac{2 m^{*} y}{\hbar^{2}}\right)^{1 / 2}\right]
\end{aligned}
$$

with $A_{n^{\prime} n}^{ \pm}(y)=\epsilon_{n}+\left(\epsilon_{n^{\prime}}-\epsilon_{n} \mp \hbar \omega_{\mathrm{LO}}+y\right)^{2} / 4 y$. Noting that the EELR is defined by $P=\operatorname{Re} \sigma E_{0}^{2}$, the EELR induced by electron-LO-phonon interactions in a 2DEG can be obtained from the energy-balance equation

$$
\begin{aligned}
\operatorname{Re} \sigma E_{0}^{2}= & \frac{2 \alpha L_{0} \omega_{\mathrm{LO}}^{3} m^{* 2}}{\pi^{3 / 2} \hbar^{2}} \sqrt{k_{B} T_{e}} \sum_{n^{\prime}, n} \int_{0}^{\infty} \frac{d y}{\sqrt{y}} \\
& \times X_{n^{\prime} n}\left[\left(\frac{2 m^{*} y}{\hbar^{2}}\right)^{1 / 2}\right]\left[\left(N_{0}+1\right) e^{-A_{n^{\prime} n}^{-}(y) / k_{B} T_{e}}\right. \\
& \left.-N_{0} e^{-A_{n^{\prime} n}^{+}(y) / k_{B} T_{e}}\right] .
\end{aligned}
$$

Equation (9) reflects the fact that the net energy transfer rate is the difference between phonon emission and absorption by heated electrons in the structure and that only inelastic scattering processes contribute to electron-energy loss and to the heating of the electrons.

Using the momentum- and energy-balance equations, we can obtain the momentum relaxation time $\tau$ and electron temperature $T_{e}$ for a given ac driving field with strength $E_{0}$ and frequency $\omega$, and from them obtain the conductivity and the EELR. To proceed, we must specify the form factor and electronic subband energy, which specify the particular 2DEG structure. In this paper, we discuss the case of an $\mathrm{Al}_{x} \mathrm{Ga}_{1-x} \mathrm{As} / \mathrm{GaAs}$ heterojunction.

\section{For the case of an $\mathrm{Al}_{x} \mathrm{Ga}_{1-x} \mathrm{As} / \mathrm{GaAs}$ heterojunction}

For a high-mobility $\mathrm{Al}_{0.3} \mathrm{Ga}_{0.7} \mathrm{As} / \mathrm{GaAs}$ heterojunction, only the lowest electronic subband is occupied by electrons when the total electron density is less than $6 \times 10^{15} \mathrm{~m}^{-2}$, and the energy spacing between the ground state and the first excited state is rather large (normally, $\epsilon_{1}-\epsilon_{0}>50 \mathrm{meV}$ ). ${ }^{18}$ The effect of thermal occupation of electrons in the higher subbands is very small because of the relatively low electron density and large energy separation between subbands. Hence we can consider the one-subband case in our calculation. In the present study we apply the usual triangular well approximation to the model the confining potential normal to the interface of the $\mathrm{Al}_{x} \mathrm{Ga}_{1-x} \mathrm{As} / \mathrm{GaAs}$ heterojunction and use the corresponding variational wave function of the ground state, ${ }^{19}$ which results in

$$
X_{00}(q)=\frac{\pi}{8 b} \frac{8+9 x+3 x^{2}}{x(1+x)^{3}}
$$

where $x=q / b$ and $b=\left[\left(48 \pi m^{*} e^{2} / \kappa \hbar^{2}\right)\left(N_{\mathrm{depl}}+11 n_{e} / 32\right)\right]^{1 / 3}$ defines the thickness $(\sim 3 / b)$ of the triangular well with $\kappa$ the dielectric constant and $N_{\text {depl }}$ being the depletion charge density. After taking into consideration the above remarks and measuring the energy from the lowest subband, i.e., taking $\epsilon_{0}=0$, the momentum- and energy-balance equations for $\mathrm{Al}_{x} \mathrm{Ga}_{1-x} \mathrm{As} / \mathrm{GaAs}$ heterojunctions are obtained, respectively, by

$$
\begin{aligned}
\frac{1}{\tau}= & \frac{\alpha \omega_{\mathrm{LO}} N_{0} e^{\beta_{e} / 2}}{32 n_{e} L_{0}^{2}}\left(\frac{\beta_{e}}{\pi}\right)^{1 / 2}\left\{e^{\left(\beta_{0}-\beta_{e}\right)}\left[R_{0}\left(\beta_{e}\right)+R_{1}\left(\beta_{e}\right)\right]\right. \\
& \left.+\left[R_{0}\left(\beta_{e}\right)-R_{1}\left(\beta_{e}\right)\right]\right\}
\end{aligned}
$$

and

$$
\frac{\omega_{\mathrm{LO}} \tau}{1+(\omega \tau)^{2}}\left(\frac{E_{0}}{F_{0}}\right)^{2}=\frac{\alpha N_{0} e^{\beta_{e} / 2} R_{1}\left(\beta_{e}\right)}{32 n_{e} L_{0}^{2} \sqrt{\pi \beta_{e}}}\left[e^{\left(\beta_{0}-\beta_{e}\right)}-1\right] .
$$

Here, $\quad \beta_{0}=\hbar \omega_{\mathrm{LO}} / k_{B} T, \quad \beta_{e}=\hbar \omega_{\mathrm{LO}} / k_{B} T_{e}, \quad F_{0}=$ $\omega_{\mathrm{LO}}\left(2 m^{*} \hbar \omega_{\mathrm{LO}}\right)^{1 / 2} / e$ is the polar-electric-field strength, and

$$
R_{j}(x)=\int_{0}^{\infty} \frac{d y}{y^{j}} \frac{8+9 z+3 z^{2}}{(1+z)^{2}} \exp \left[-\frac{x}{4}\left(y+\frac{1}{y}\right)\right],
$$

with $z=\sqrt{y} / b L_{0}$. From the energy-balance equation [Eq. (12)], we note that the EELR is zero when $T_{e} \rightarrow T$. Now we have obtained the simple formulas to calculate $\tau$ and $T_{e}$ as a function of $E_{0}$ and $\omega$ in 2DSS's such as $\mathrm{Al}_{x} \mathrm{Ga}_{1-x} \mathrm{As} / \mathrm{GaAs}$ heterojunctions.

\section{RESULTS AND DISCUSSIONS}

The numerical results of this paper pertain to $\mathrm{Al}_{x} \mathrm{Ga}_{1-x} \mathrm{As} / \mathrm{GaAs}$ heterojunctions and the material parameters for $\mathrm{GaAs}$ are taken as (1) effective-electron-mass ratio $m^{*} / m_{e}=0.0665$ with $m_{e}$ the electron rest mass; (2) static dielectric constant $\kappa=12.9$; (3) electron-LO-phonon coupling constant $\alpha=0.068$; and (4) LO-phonon energy $\hbar \omega_{\mathrm{LO}}=36.6 \mathrm{meV}$. In the calculations, we use a typical electron density $n_{e}=2 \times 10^{15} \mathrm{~m}^{-2}$ and a typical depletion charge density $N_{\text {depl }}=5 \times 10^{14} \mathrm{~m}^{-2}$ for an $\mathrm{Al}_{x} \mathrm{Ga}_{1-x} \mathrm{As} / \mathrm{GaAs}$ heterostructure.

The electron temperature $T_{e}$, inverse of the momentum relaxation time $\tau^{-1}$, and real part of the conductivity $\operatorname{Re} \sigma$ as a function of radiation intensity (i.e., ac electric field strength, $\left.E_{0}\right)$ are shown in Fig. 1 for different radiation frequencies (i.e., ac electric field frequencies, $\omega$ 's) at a fixed sample temperature $T$. At lower driving fields, the electron temperature (the momentum relaxation time) is very close to sample temperature (to its value at zero frequency) and a linear response is evident [see Fig. 1(c)]. The influence of the radiation frequency is very weak on $T_{e}$ and $\tau$ when $E_{0}$ is small, in contrast to a strong dependence of conductivity on the frequency. With increasing radiation intensity $E_{0}$, (1) the electrons are heated and the electron temperature increases rapidly; (2) $T_{e}>T$ can be seen for all frequencies; (3) the inverse of the relaxation time, which corresponds to the scattering rate, increases due to the rise of $T_{e}$; and (4) the conductivity first increases and then decreases when $\omega \neq 0$. The radiation frequency plays an important role in observing these phenomena. The higher the frequency is, the stronger the driving field required to heat the electrons and to enhance the scattering rate. The conductivity (or mobility) decreases with increasing $\omega$ at a fixed $E_{0}$. However, when the driving field is large enough $\left(E_{0}>50 \mathrm{kV} / \mathrm{cm}\right.$ at $\left.T=77 \mathrm{~K}\right)$, a rather weak effect of $\omega$ on $T_{e}, \tau$, and $\operatorname{Re} \sigma$ can be found. From the results shown in Fig. 1(b), we note that under the intense $\mathrm{THz}$ driving, the scattering rate (or inverse of the relaxation time $\tau^{-1}$ ) induced by electron-LO-phonon interactions may 

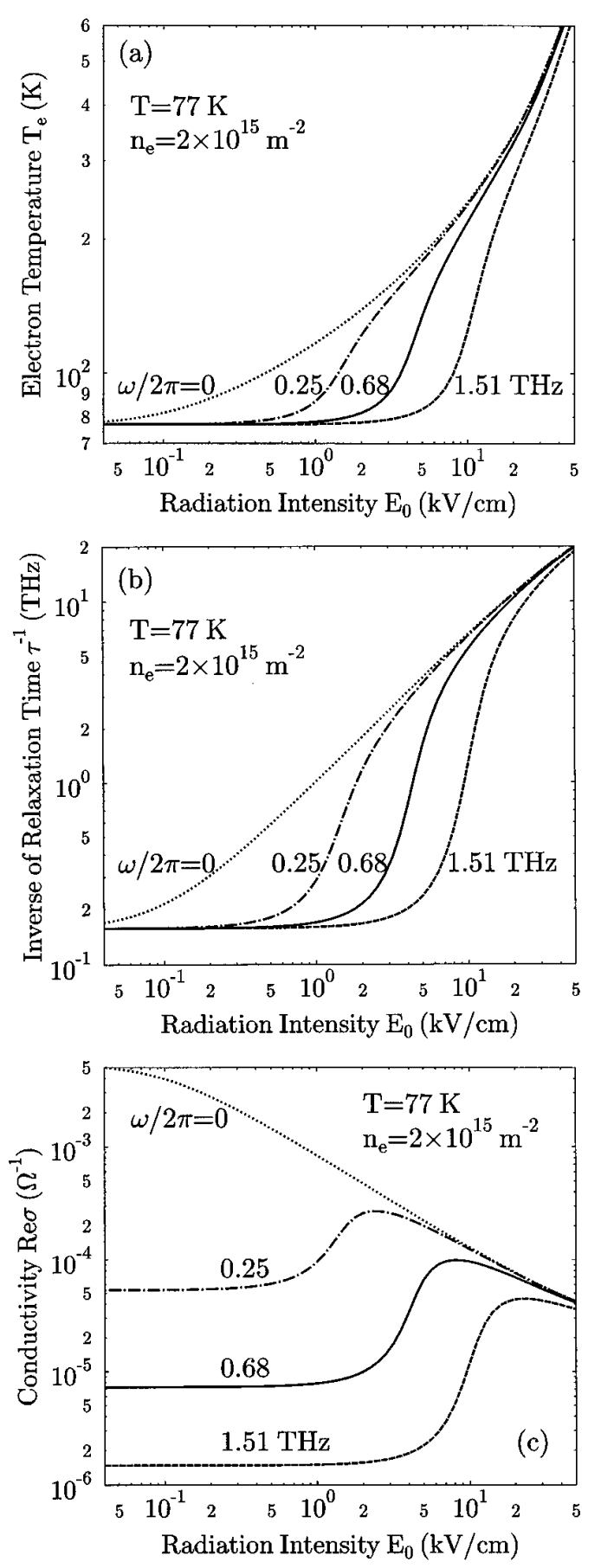

FIG. 1. Dependence of (a) electron temperature $T_{e}$, (b) inverse of momentum relaxation time $\tau^{-1}$, and (c) real part of conductivity $\operatorname{Re} \sigma$ for an $\mathrm{Al}_{x} \mathrm{Ga}_{1-x} \mathrm{As} / \mathrm{GaAs}$ heterojunction on radiation intensity $E_{0}$ for different radiation frequencies $\omega$ at a fixed sample temperature $T$. $\omega=0$ corresponds to a dc electric field.

be on the scale of the radiation frequency so that the radiation may modify strongly the process of momentum and energy relaxation of the excited electrons in the structure. This is the main physical reason why the interesting features of nonlinear transport and optical phenomena can be observed in an $\mathrm{Al}_{x} \mathrm{Ga}_{1-x} \mathrm{As} / \mathrm{GaAs}$-based 2DSS driven by intense $\mathrm{THz}$ electromagnetic fields. In our calculations, we have taken the strength and frequency of the $\mathrm{THz}$ electromagnetic field as

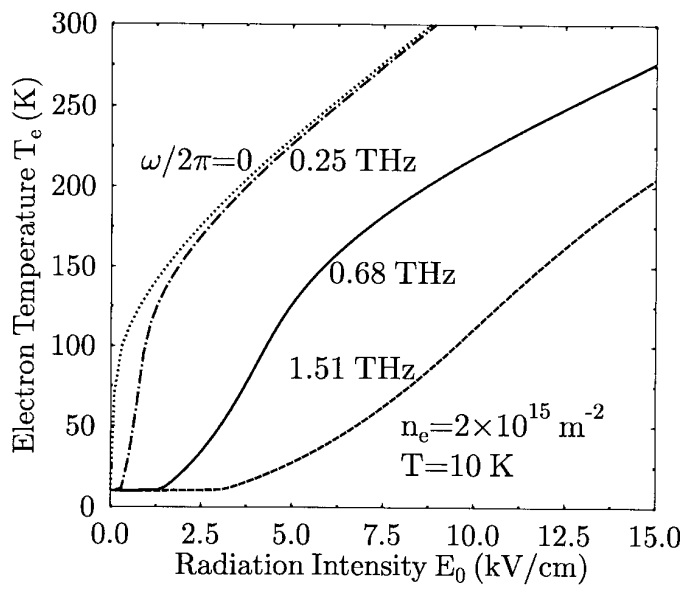

FIG. 2. Electron temperature $T_{e}$ in an $\mathrm{Al}_{x} \mathrm{Ga}_{1-x} \mathrm{As} / \mathrm{GaAs}$ heterojunction as a function of radiation intensity $E_{0}$ at a fixed sample temperature $T=10 \mathrm{~K}$ for different radiation frequencies.

those used in the experimental studies in Ref. 4.

The results obtained from our model calculation and shown in Fig. 1 indicate that a THz-frequency-modified nonlinear effect is not pronounced at very high driving fields. When $E_{0}$ is very large, $T_{e} \gg T$ which leads to $\tau^{-1} \gg \omega$ and $\omega \tau \ll 1$ so that the effect of $\omega$ is suppressed. We would like to point out that in our model we did not include effects such as electron-electron interactions, hot-phonon effects, nonparabolicity of the conduction-band structure, etc. These effects are very important in studying the transport and optical properties at very high fields.

In Fig. 2 we plot the electron temperature as a function of radiation intensity at a fixed sample temperature $T=10 \mathrm{~K}$ for different radiation frequencies: $\omega / 2 \pi=0,0.25,0.68$, and 1.51 THz. These are the conditions for the measurements conducted in Ref. 4. Now, we compare the results obtained from this simple model calculation with the experimental data shown in Fig. 4 of Ref. 4. The results shown in Fig. 2 indicate that (i) at a fixed ac field strength, electron temperature decreases with increasing frequency. This agrees with the data shown in Figs. 4(b) and 4(c) of Ref. 4 over a field range $0<E_{0}<15 \mathrm{kV} / \mathrm{cm}$. (ii) Generally, $T_{e}$ does not increase linearly with $E_{0}$. It implies that the theoretical results are closer to the data obtained from PL measurements in Ref. 4, especially for relatively high driving fields and high frequencies (e.g., at $1.51 \mathrm{THz}$ and $E_{0}>6 \mathrm{kV} / \mathrm{cm}$ ) where a dispersion can be found for the data obtained from PL and transport measurements. (iii) At relatively low fields and low frequencies, for example when $E_{0}<0.6 \mathrm{kV} / \mathrm{cm}$ at $\omega / 2 \pi=0.25 \mathrm{THz}$, a roughly linear increase in $T_{e}$ with $E_{0}$ may be drawn. This is in line with experiments. (iv) Quantitatively, our results agree with the experimental data, especially with those obtained from PL measurements. From Fig. 2 shown here and Fig. 4 shown in Ref. 4 we see that at $T=10 \mathrm{~K}, T_{e}$ varies from 10 to about $100 \mathrm{~K}(300 \mathrm{~K}, 200 \mathrm{~K})$ when $E_{0}$ is increased from 0 to about $0.6 \mathrm{kV} / \mathrm{cm}(15 \mathrm{kV} / \mathrm{cm})$ at $\omega / 2 \pi=0.25 \mathrm{THz}$ $(0.68 \mathrm{THz}, 1.51 \mathrm{THz})$. The theoretical results presented in this paper confirm the main conclusions drawn from the experimental measurements in Ref. 4, that is, (1) the electronenergy distribution can be characterized by a statistical distribution function with an effective electron temperature; (2) 


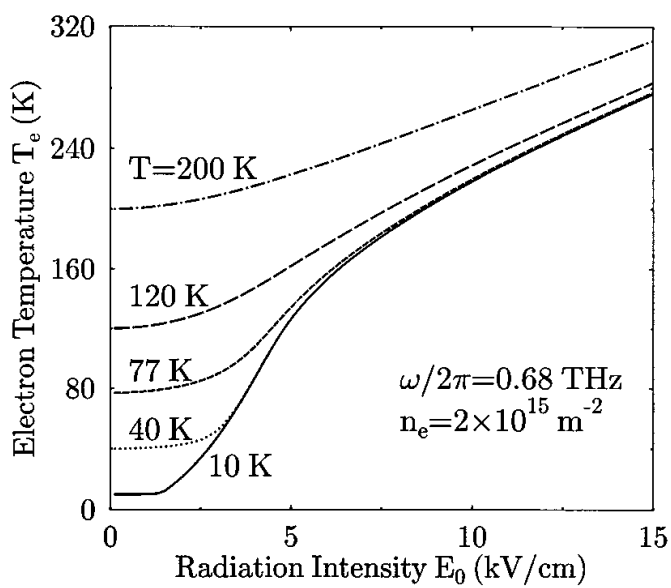

FIG. 3. Electron temperature as a function of radiation intensity at a fixed radiation frequency for different sample temperatures.

under intense $\mathrm{THz}$ drive, the electron-LO-phonon scattering dominates both momentum and energy relaxation for excited electrons in $\mathrm{Al}_{x} \mathrm{Ga}_{1-x} \mathrm{As} / \mathrm{GaAs}$-based 2DSS's. This occurs even at relatively low sample temperatures, e.g., at $T=10 \mathrm{~K}$; and (3) over a driving field range $0<E_{0}<15 \mathrm{kV} / \mathrm{cm}$, the electron distribution maintains a heated thermal form and the hot-phonon effects may be negligible.

The influence of sample temperature on the heating of electrons can be seen from Fig. 3. Under the action of the driving ac and/or dc fields, the electrons in the system can gain the energy from the field applied and from the phonon absorption scattering process and lose the energy through emitting phonons. This process has been reflected by the energy-balance equation. At a low $T$, for example at $T=10$ $\mathrm{K}$, a changeover from linear response (i.e., $T_{e} \sim T$ ) to nonlinear response (i.e., $T_{e}>T$ ) with increasing field can be more clearly seen. At a sufficiently low sample temperature, the electrons gain energy mainly from the applied field because of the small LO-phonon occupation number so that the effect of LO-phonon absorption is very weak. At sufficiently low fields, the relaxation time for electrons is rather long [see Fig. 1(b)] so that an electron has enough time to get the energy $E \sim \hbar \omega_{\mathrm{LO}}$ from the field and then lose it by LOphonon emission. This two-step process is analogous to elastic scattering which will not result in energy loss and, consequently, in the heating of the electrons. At sufficiently high fields, an electron can be accelerated quickly to reach an energy level $E \geqslant \hbar \omega_{\mathrm{LO}}$. When the time of this process required is shorter than the relaxation time, electrons will be heated by the electric field and will enter the nonlinear response regime. The electrons can be heated more efficiently at a lower sample temperature than at a higher sample temperature, due to weaker LO-phonon absorption scattering at a lower $T$. It implies an enhancement of the net energy transfer rate at low $T$ 's. With increasing sample temperature, the increase in LO-phonon absorption scattering will lead to a smear on these processes, and so (1) the demarcation between a linear and nonlinear response cannot be clearly distinguished; and (2) the electrons are less efficiently heated at high fields.

\section{SUMMARY}

In this paper we have studied theoretically the nonlinear electron transport in two-dimensional semiconductor systems in intense $\mathrm{THz}$ electromagnetic fields. The prime motivation of this study is recent experiments ${ }^{4}$ carried out using the free-electron lasers. We have developed a model which can be applied to the study of nonlinear transport and optical properties in THz-driven 2DEG's. To understand the most important physical mechanism responsible for the experimental findings, we considered a simple case where only the electron-LO-phonon interactions are taken into account under the lowest-order approximation and effects such as electron-electron interactions, direct electron-photon interactions, higher-order electron-photon-phonon interactions, hotphonon effects, etc. are not included in our model. We also made our model calculation rather simple, through (i) applying the balance equation approach to the steady-state Boltzmann equation; (ii) using a drifted Maxwellian as the electron distribution function, where an electron temperature $T_{e}$ is introduced to describe the thermal term of the EDF; (iii) neglecting the contributions from higher orders of electron velocity, where we assume $\hbar v k_{x} \ll k_{B} T_{e}$; and (iv) considering only the momentum- and energy-balance equations. Further, a situation in conjunction with experiments in Ref. 4 has been taken into account, that is, (1) the strength of the ac electric field provided by a FEL is much larger than that of the dc field used in the transport measurements; and (2) the $\mathrm{THz}$ electromagnetic radiations are polarized along the $2 \mathrm{D}$ plane of a 2DSS. Using the momentum- and energy-balance equations developed in this paper, one can determine the electron temperature and momentum relaxation time for a given ac driving field with a strength $E_{0}$ and frequency $\omega$, and from them obtain quantities such as conductivity and electron-energy-loss rate.

For the case of an $\mathrm{Al}_{x} \mathrm{Ga}_{1-x} \mathrm{As} / \mathrm{GaAs}$ heterostructure, we have studied the dependence of the electron temperature, momentum relaxation time, and real part of the conductivity on the $\mathrm{THz}$ drive amplitude for different $\mathrm{THz}$ frequencies and sample temperatures. The results obtained from this study were found to be in line with those reported in Ref. 4. We also presented a detailed discussion and analysis of the nonlinear transport phenomena observed from this study. The most significant conclusion we draw from our study is that under the intense $\mathrm{THz}$ electromagnetic radiation, the scattering rate or inverse of the relaxation time induced by electron-LO-phonon interactions in an $\mathrm{Al}_{x} \mathrm{Ga}_{1-x} \mathrm{As} / \mathrm{GaAs}-$ based 2DSS may be on the scale of the radiation frequency so that the radiation may modify strongly the processes of momentum and energy relaxation of the excited electrons in the structure. This is the main physical reason some interesting features of nonlinear transport and optical phenomena can be observed in such systems in strong $\mathrm{THz}$ electromagnetic fields. Our results indicated that the frequency and intensity of $\mathrm{THz}$ radiations used in the measurements conducted in Ref. 4 were located in a regime where the strongest nonlinear effects can be observed for the chosen sample temperatures.

Finally, some of the approximations we made in the present model calculation were based only on current experimental results and experimental conditions. The investiga- 
tion of transport and optical properties in THz-driven 2DEG's is a fast-growing research field which is very rich in physics. By going beyond these approximations, one may predicate some new phenomena theoretically, such as an anisotropic conductivity induced by an anisotropic electronic transition rate, nonequilibrium behaviours induced by the electron occupation to the excited states, phonon-assisted multiphoton absorptions, etc. These effects need experimental verification.

\section{ACKNOWLEDGMENT}

This work was supported by the Australian Research Council.
*Electronic mail: xu@feynman.sci.uow.edu.au

${ }^{1}$ W. Xu and C. Zhang, Phys. Rev. B 54, 4907 (1996).

${ }^{2}$ W. Xu and C. Zhang, Appl. Phys. Lett. 68, 3305 (1996).

${ }^{3}$ W. Xu, F. M. Peeters, and J. T. Devreese, Phys. Rev. B 48, 1562 (1993).

${ }^{4}$ N. G. Asmar, J. Cerne, A. G. Markelz, E. G. Gwinn, M. S. Sherwin, K. L. Campman, and A. C. Gossard, Appl. Phys. Lett. 68, 829 (1996).

${ }^{5}$ N. G. Asmar, A. G. Markelz, E. G. Gwinn, J. Cerne, M. S. Sherwin, K. L. Campman, P. F. Hopkins, and A. C. Gossard, Phys. Rev. B 51, 18041 (1995).

${ }^{6}$ N. Tzoar, P. M. Platzman, and A. Simons, Phys. Rev. Lett. 36, 1200 (1976), and references for their early work up to 1963 therein.

${ }^{7}$ W. Götze and P. Wölfle, Phys. Rev. B 6, 1226 (1972).

${ }^{8}$ N. Tzoar and Chao Zhang, Phys. Rev. B 35, 7596 (1987).
${ }^{9}$ T. Brandes, Europhys. Lett. 33, 629 (1996).

${ }^{10}$ Z. Q. Zou and X. L. Lei, Phys. Rev. B 51, 9493 (1995), and references for the early work by X. L. Lei et al. therein.

${ }^{11}$ X. L. Lei and N. J. M. Horing, Phys. Rev. B 36, 4238 (1987).

${ }^{12}$ S. K. Ma and K. W. -K. Shung, Phys. Rev. B 48, 10751 (1993).

${ }^{13}$ W. Cai, P. Hu, T. F. Zheng, B. Yudanin, and M. Lax, Phys. Rev. B 40, 7671 (1989), and references for their early work therein.

${ }^{14}$ K. K. Thornber and P. R. Feynman, Phys. Rev. B 1, 4099 (1970).

${ }^{15}$ F. M. Peeters and J. T. Devreese, Phys. Rev. B 23, 1936 (1981).

${ }^{16}$ X. L. Lei and C. S. Ting, Phys. Rev. B 30, 4809 (1984).

${ }^{17}$ W. Xu, F. M. Peeters, and J. T. Devreese, Phys. Rev. B 43, 14134 (1991); Semicond. Sci. Technol. 7, 1251 (1992).

${ }^{18}$ R. Fletcher, E. Zaremba, M. D'Iorio, C. T. Foxon, and J. J. Harris, Phys. Rev. B 41, 10649 (1990).

${ }^{19}$ T. Ando, A. B. Fowler, and F. Stern, Rev. Mod. Phys. 54, 437 (1982). 